\title{
On the Computation of Debye Functions of Integer Orders
}

\author{
By E. W. Ng and C. J. Devine
}

\begin{abstract}
An efficient method is presented for the computation of Debye functions of integer orders to twenty significant decimal digits.
\end{abstract}

The Debye functions occur in thermodynamic problems, for example, in the context of crystallographic structure or that of radiation. They are sometimes labeled radiation integrals. For a recent application, refer to Howard and Grindlay [1], and for a survey of numerical tables refer to Fletcher et al. [2]. We shall define these functions as:

$$
\bar{D}_{p}(x)=\frac{1}{\Gamma(p+1)} \int_{0}^{x} \frac{t^{p}}{e^{t}-1} d t, \quad R(x) \geqq 0,
$$

and

$$
D_{p}(x)=\frac{1}{\Gamma(p+1)} \int_{x}^{\infty} \frac{t^{p}}{e^{t}-1} d t
$$

where $\bar{D}_{p}(x)+D_{p}(x)=\zeta(p+1)$, Riemann's zeta function. Thus the Debye functions are essentially incomplete Riemann zeta functions. Recently Howard and Grindlay [1] used these functions for $p=0.5(0.5) 2.5$ in the solution of a transcendental equation.

Y. L. Luke $\lceil 3\rceil$ studied the function $A(x, m)=\left(m m ! / x^{m}\right) \bar{D}_{m}(x)$ for $x$ complex and $m$ an integer. He presented approximations based on the Padé approximation for $t /\left(e^{t}-1\right)$, and provided numerical examples for $x$ real, $0 \leqq x \leqq 10$, and $m=1(1) 4$. He further showed that approximations for higher $m$ can be generated by use of simple recurrence formulas. It should also be observed that, using the basic data given by Luke, it is straightforward to derive approximations for values of $m$ other than an integer, say $m$ an odd multiple of $\frac{1}{2}$.

In this note we suggest an alternative method for computing $\bar{D}_{m}(x)$ and $D_{m}(x)$ for $m=1(1) 10$ and $0 \leqq(\operatorname{Real} x) \leqq \infty$, to an accuracy of twenty significant figures.

Consider the Bose-Einstein functions

$$
B_{p}(\eta, x)=\frac{1}{\Gamma(p+1)} \int_{0}^{x} \frac{t^{p} d t}{e^{t-\eta}-1}
$$

and

Received June 23, 1969.

AMS Subject Classifications. Primary 6525, 3345; Secondary 4130.

Key Words and Phrases. Debye functions, Riemann zeta functions, chemical physics. 


$$
B_{p}(\eta)=\lim _{x \rightarrow \infty} B_{p}(\eta, x)
$$

It is apparent that $\bar{D}_{p}(x)=B_{p}(0, x)$. Furthermore, by a simple change of variable, we obtain

$$
D_{p}(x)=\frac{1}{\Gamma(p+1)} \int_{0}^{\infty} \frac{(y+x)^{p} d y}{e^{y+x}-1}, \quad y \geqq 0 .
$$

For $p$ an integer $m,(5)$ is readily reduced to a linear combination of $B_{k}(x)$, i.e.

$$
x^{-m} D_{m}(x)=\sum_{k=0}^{m} \frac{x^{-k}}{(m-k) !} B_{k}(-x), \quad 0 \leqq x \leqq \infty .
$$

Notice that if we expand the function $B_{k}(-x)$ in powers of $e^{-x}[4]$, then Eq. (6) is exactly equivalent to the series representation for $D_{m}(x)$ given by Stegun [5, p. 998]. Recently the authors [4] produced Chebyshev expansions for the functions $B_{k}(x)$, $k=1(1) 10$ with $x$ in the closed intervals $[-\infty,-1]$ and $[-1,1] . B_{k}(x)$ in the interval $[1, \infty]$ is simply related to $B_{k}(-x)$ and thus readily computable. These expansions are very efficient, each having 10 to 20 terms for 20 decimal accuracy. Thus, if one wants to compute a sequence of $D_{k}(x), k=1,2, \ldots, m$, Eq. (6) together with the expansions in [4] would serve as a good algorithm, since one can first compute a sequence of values $B_{k}(-x), k=1,2, \ldots, m$ and then form the linear combinations. A still better algorithm would be to use Eq. (6) for $D_{m}(x)$ in $[1, \infty]$ and the Taylor series for $\bar{D}_{m}(x)$ in $[0,1]$, which is

$$
\begin{aligned}
\bar{D}_{m}(x) & =x^{m} \sum_{k=0}^{\infty} \frac{B_{k} x^{k}}{k !(m+k)} \\
& =x^{m}\left[\frac{1}{m}-\frac{x}{2(m+1)}+\frac{x^{2}}{2 \pi^{2}} \sum_{k=0}^{\infty}(-1)^{k} \frac{\zeta(2 k+2)}{2 k+m+2}\left(\frac{x}{2 \pi}\right)^{2 k}\right] .
\end{aligned}
$$

In the above expansions $B_{k}$ is Bernoulli's number and $\zeta$ is Riemann's zeta function. For the interval $[0,1]$ expansion (8) converges rapidly and is suitable for computation, as evidenced by the following truncation error bounds:

\begin{tabular}{cc}
\hline$k$ & Maximum Absolute Error on Truncation after $(k)$ th Term \\
\hline 10 & $6 \times 10^{-21}$ \\
15 & $5 \times 10^{-29}$ \\
20 & $4 \times 10^{-37}$ \\
\hline
\end{tabular}

We are indebted to the referee for helpful comments. 
1. R. Howard \& J. Grindlay, "Tables of Debye functions," Canad. J. Phys., v. 44, 1966, pp. 45-56. MR 32 \# 3768.

2. A. Fletcher, et AL, An Index of Mathematical Tables. Vol. I: Introduction, Addison-Wesley, Reading, Mass., 1962, p. 543. MR 26 \#365a.

3. Y. L. Luke, The Special Functions and Their Approximations, Vol. 1 \& 2, Academic Press, New York, 1968.

4. E. W. Ng \& C. J. Devine, "Chebyshev polynomial expansion of Bose-Einstein functions of orders 1 to 10," Math. Comp., v. 23, 1969, pp. 639-643

5. M. Abramowitz \& I. A. STegun, Editors, Handbook of Mathematical Functions with Formulas, Graphs and Mathematical Tables, Nat. Bur. Standards Appl. Math. Series, 55, Superintendent of Documents, U.S. Government Printing Office, Washington, D.C., 1964, 3rd printing with corrections, 1965. MR 29 \# 4914; MR 31 \# 1400. 